\title{
Individualised behaviour change strategies for physical activity in multiple sclerosis (IPAC-MS): protocol for a randomised controlled trial
}

\author{
Farren L. Goulding ${ }^{1}$, Charity D. Evans ${ }^{1 *}$ (D, Katherine B. Knox², Hyun J. Lim², Michael C. Levin² and Sarah J. Donkers²
}

\begin{abstract}
Background: Multiple sclerosis (MS) is a chronic, degenerative disease of the central nervous system. Because of the long-term and unpredictable nature of the disease, the burden of MS is significant from both a patient and societal perspective. Despite a recent influx of disease-modifying therapies to treat MS, many individuals continue to experience disability that negatively affects productivity and quality of life. Previous research indicates that physical activity has a positive impact on walking function in individuals with MS, in addition to the usual beneficial effects on overall health. However, most people with MS are not active enough to gain these benefits, and a lack of support to initiate and maintain physical activity has been identified as a major barrier. This study will evaluate the impact of a novel intervention involving individualised behaviour change strategies delivered by neurophysiotherapists on increasing physical activity levels in individuals with MS who are currently inactive.

Methods/design: This single-blind, parallel-group, randomised controlled trial will be conducted in Saskatchewan, Canada. Eligible participants include individuals with MS who are ambulatory but identified as currently inactive by the self-reported Godin Leisure-Time Exercise Questionnaire (GLTEQ). The intervention will be delivered by neurophysiotherapists and includes individualised behaviour change strategies aimed at increasing physical activity over a 12-month period. The control group will receive usual care during the 12-month study period. The primary outcome is the change in physical activity level, as measured by the change in the GLTEQ score from baseline to 12 months. Secondary outcomes include the change in patient-reported outcome measures assessing MS-specific symptoms, confidence and quality of life.
\end{abstract}

Discussion: Physical activity has been identified as a top research priority by the MS community. Findings from this novel study may result in new knowledge that could significantly impact the management and overall health of individuals with MS.

Trial registration: ClinicalTrials.gov, NCT04027114. Registered on 10 July 2019.

Keywords: Multiple sclerosis, Exercise, Physical activity, Individualised, Behaviour change strategy, Neurophysiotherapist, Randomised controlled trial

\footnotetext{
* Correspondence: charity.evans@usask.ca

${ }^{1}$ College of Pharmacy and Nutrition, University of Saskatchewan, 104 Clinic

Place, Saskatoon, SK, Canada

Full list of author information is available at the end of the article
}

(c) The Author(s). 2019 Open Access This article is distributed under the terms of the Creative Commons Attribution 4.0 International License (http://creativecommons.org/licenses/by/4.0/), which permits unrestricted use, distribution, and reproduction in any medium, provided you give appropriate credit to the original author(s) and the source, provide a link to the Creative Commons license, and indicate if changes were made. The Creative Commons Public Domain Dedication waiver (http://creativecommons.org/publicdomain/zero/1.0/) applies to the data made available in this article, unless otherwise stated. 


\section{Introduction}

Multiple sclerosis (MS) is a chronic degenerative neurological disease involving the central nervous system. Symptoms of MS are unpredictable and can affect multiple body systems. The disease may be broadly categorised as relapsing-remitting or progressive [1]. Over time, most cases follow a progressive course [2], and an estimated $50 \%$ of individuals with MS require a cane within 15 years of disease onset [3]. There is no cure for MS, and although several different disease-modifying therapies are available, there is still controversy about their long-term effectiveness, and they are not indicated for all individuals with MS [4].

In the general population, people who are moderately physically active have a lower risk for medical comorbidities and an increase in lifespan by an average of 7 years [5]. Physical activity has been shown to have considerable benefit in MS, regardless of disease type or duration [6]. Physical activity has also been proposed to have a disease-modifying impact [7], supported by magnetic resonance imaging outcomes [8] and research in animal models [9]. Regardless of evidence supporting a positive effect, less than $20 \%$ of individuals with MS are sufficiently active for health benefits [10]. Despite the known benefits of exercise in MS, there has been little change reported in physical activity levels in the MS population over the past 25 years [11]. Historically, the standard approach for promoting physical activity in MS research has involved structured exercise training [12]. However, individuals with MS routinely cite a lack of support and resources as a major barrier to regular physical activity [5]. A recent review on exercise interventions in MS identified the largest effect sizes for increasing physical activity were from those involving behaviour change strategies [13]. The same review noted that existing behaviour change strategy research was difficult to replicate and implement in clinical practice due to insufficient detail about the actual interventions [13].

In order to better describe the active components of behaviour change interventions and adopt the most effective behaviour change strategies, the Behavior Change Technique Taxonomy (BCTT) was created [14]. The BCTT includes a comprehensive list and definitions of behaviour change strategies. Behavior Change Theory describes the 'why' and informs selection of behaviour change strategies, or the 'how' $[15,16]$. Few studies have applied theories of behaviour change in MS physical activity interventions [17], and those that have were focused largely on 'packaged' rather than individualised behaviour change interventions [18]. For example, fatigue in MS is identified as a major barrier to exercise [19]. A study may deliver a behaviour change intervention through a well-designed fatigue self-management programme; however, not every individual with MS will have fatigue as their main barrier to physical activity. Therefore, individualised behaviour change strategies that address each person's unique and most significant barriers are recommended; yet, few have been applied in MS physical activity research to date [20].

Although effective at increasing physical activity behaviour, behaviour change strategies alone are believed to account for only $20 \%$ of change [16]. A recent study on behaviour change interventions recommended the added value of professional support [21], because individuals with MS benefit from the intermittent support of a specialist with expertise in exercise and MS to help maintain activity levels and function as the disease progresses [22]. The purpose of this study is to evaluate if a novel intervention of individualised behaviour change strategies delivered by neurophysiotherapists with expertise in MS increases physical activity levels in individuals with MS who are currently inactive.

\section{Methods/design \\ Study design and setting}

The IPAC-MS (Individualized Physiotherapy and Activity Coaching for Multiple Sclerosis) study is a prospective, single-blind, parallel-group, randomised controlled trial conducted in the Canadian province of Saskatchewan. The study is designed as a superiority trial and is a collaborative effort of interdisciplinary researchers, clinicians, and patient and family advisors Additional file 1.

\section{Study participants}

Participants will be recruited primarily through the Saskatchewan MS Drugs Program (SMSDP). The SMSDP is a provincial initiative created to oversee the applications of all individuals applying for government coverage of a disease-modifying therapy for MS. At the time of enrolment in the SMSDP, individuals are offered the chance to consent to be contacted about participating in future MS-related research; those who consent are also asked to complete the Godin Leisure-Time Exercise Questionnaire (GLTEQ) [23-25]. Approximately 50\% of all applicants to the SMSDP have consented and completed the GLTEQ. Individuals with a GLTEQ score < 24 are considered not sufficiently active for substantial health benefits and will be the primary cohort targeted for recruitment. If necessary, recruitment may also occur through the Saskatoon MS Clinic, the primary referral site for all Saskatchewan patients with MS, and through local MS Society organisations.

Individuals older than 18 years of age, with clinically definite MS, having a Patient Determined Disease Steps score $\leq 6$ (i.e., able to walk with or without aids) [26], and who are estimated not to be sufficiently active for substantial health benefits (i.e., self-reported exercise less than four times weekly) are eligible for this study. Those 
who are unable to provide consent or are deemed to have a moderate-high risk for exercise-related harm based on the Physical Activity Readiness Questionnaire [27] will be excluded.

\section{Randomisation and blinding}

All consenting participants who have completed a baseline assessment will be stratified into one of three categories based on their baseline GLTEQ score $(<9,9-17$, $\geq 18$ ). Participants from each of the three strata will be randomly assigned to either the intervention or control group by a centralised telephone request to a study coordinator not involved in data collection or analyses. Randomisation lists were computer-generated in blocks of 4 to help achieve balance in the groups. The randomisation list was created prior to participant recruitment by a research team member not involved in data collection or analyses. Given the nature of the intervention, only the outcome assessors involved in the data collection will be blinded to the study group.

\section{Intervention and control}

The intervention is an individualised physical activity behaviour change programme. The intervention will be delivered by neurophysiotherapists with expertise in MS over a 12-month period. Because each programme is specifically created for each individual, components of the intervention may vary between participants. However, there are three consistent features: behaviour change strategies, recommendations for physical activity, and ongoing neurophysiotherapist support. After randomisation, participants in the intervention group will undergo a tailored intake by a neurophysiotherapist, which will serve as the foundation for the individualised approach. At the initial intake, neurophysiotherapists will evaluate the participants' individual attributes and physical activity needs in addition to a general physiotherapy assessment (e.g., MS symptoms and functional levels) to create personalised programmes. This initial intake may occur over one to three sessions and in variable formats, depending on the participants' needs. To promote consistency, neurophysiotherapists will be trained in the Behaviour Change Wheel [28], the BCTT [14], and the Social Cognitive Theory of Behaviour Change, including correlates and determinants of physical activity behaviour in MS [20]. All training of the neurophysiotherapists was done with a standardised programme and delivered by the same instructor.

All physical activity recommendations made by the neurophysiotherapists will be based on established guidelines, existing resources, and individual participant needs. Best practice guidelines recommend that individuals with MS who have mild to moderate disability work up to at least 30 minutes of moderate intensity aerobic activity twice per week and resistance exercises for major muscle groups twice per week [29]. Support from a neurophysiotherapist will be available to the intervention group throughout the study period. We have allotted 15 neurophysiotherapist contact hours per participant, and each encounter will be recorded (method of contact, service delivered, and time required) using standardised data collection forms. The neurophysiotherapist support may occur in person, via telephone, using web-based methods and/or by telehealth. The neurophysiotherapist will record the types of behaviour change strategies used with each participant according to the framework and descriptors from the BCTT [14]. Intervention participants will also receive printed educational material from the MS Society of Canada on physical activity [29], diet [30] and stress management [31] at 2, 4 and 8 months. Because participants are involved in developing their own treatment plans, good adherence to the intervention is expected.

The control group will receive the same printed educational material as the intervention group at 2, 4 and 8 months. Participants in the control group will not receive any individualised assessments or recommendations, nor will they have access to neurophysiotherapist support throughout the 12-month study period (Fig. 1). At the end of the study, the control group participants will have the opportunity to receive the intervention; however, any results generated will not be part of the primary study analyses.

\section{Study outcomes}

The primary outcome is the change in physical activity level, as measured by the change in the GLTEQ score from baseline to 12 months. The GLTEQ is a validated self-reported measure of physical activity with sensitivity to detect change in MS exercise interventions [23, 24]. The GLTEQ requests activity levels in the week prior (minimising recall bias), is easy to administer, demonstrates good test-retest reliability in ambulatory individuals with MS, and correlates with other more costly and/or less convenient measures of physical activity in MS, such as accelerometers [32]. The total GLTEQ score is calculated according to the number of self-reported strenuous, moderate- or mild-intensity physical activity in 15-minute time blocks over the week. A total leisure activity score is then computed, which includes a correction for the estimated metabolic demands associated with these intensity levels. The total GLTEQ score (range, 0-119) is recommended for use in physical activity research in MS [25], with higher scores indicating greater activity levels. A score $>23$ is considered sufficiently active for substantial health benefits [23, 25].

Secondary outcomes include the change in patientreported outcome measures assessing MS-specific 


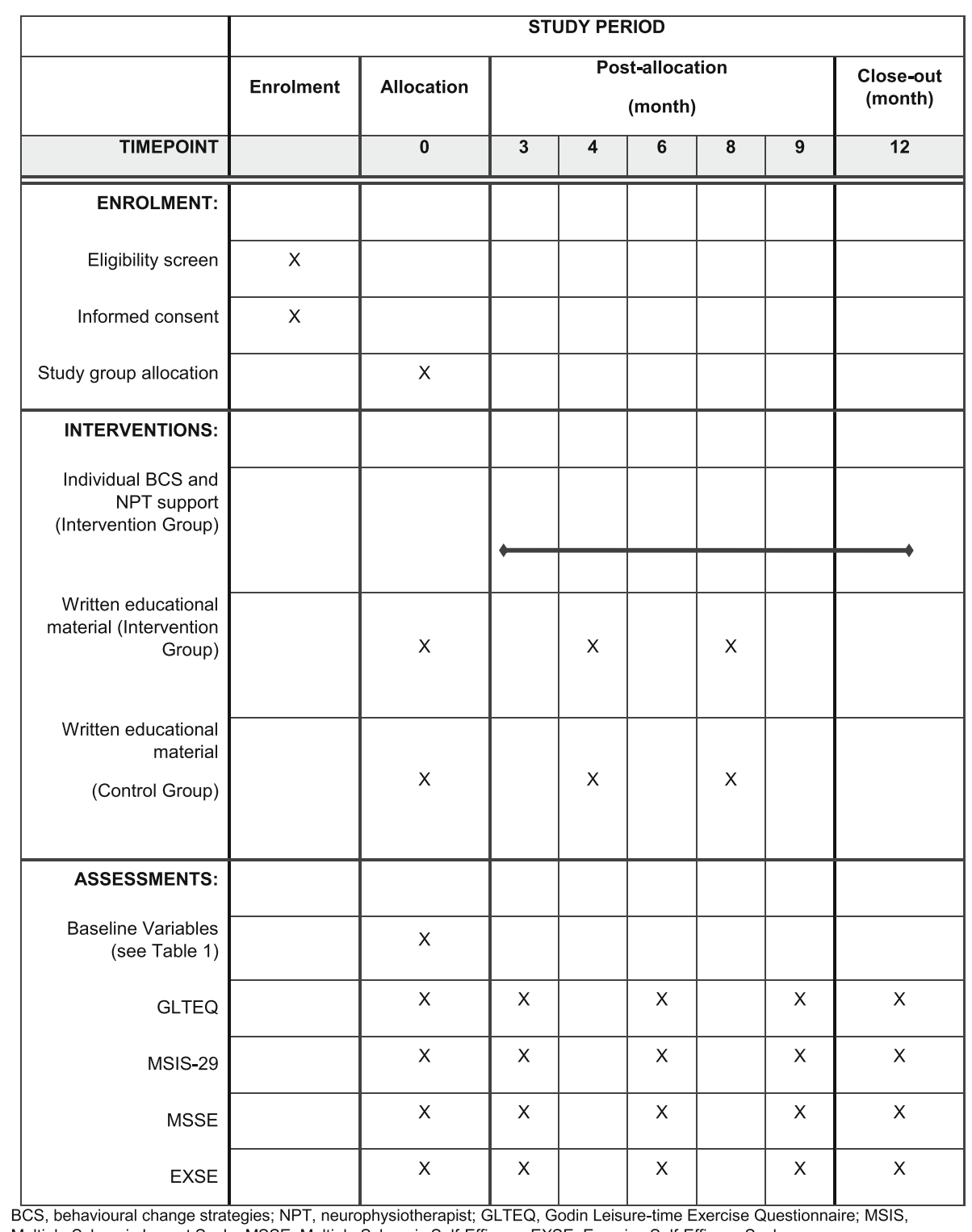
Multiple Sclerosis Impact Scale; MSSE, Multiple Sclerosis Self-Efficacy; EXSE, Exercise Self-Efficacy Scale

Fig. 1 Schedule of study enrolment, interventions, and assessments

symptoms, confidence and quality of life. The Multiple Sclerosis Impact Scale v2 [33] is a self-administered questionnaire evaluating patient-perceived physical and psychological impact of their MS. The Multiple Sclerosis Self-Efficacy Scale is an MS-specific, self-reported, selfefficacy measure with strong psychometric properties [34]. The 18-item version will be used whereby participants rate their level of confidence regarding components of disease management using a 10-point scale (very uncertain to very certain). Self-efficacy has been identified as one of the most consistent correlates of physical activity [35]. The Exercise Self-Efficacy Scale [36] is a validated and reliable measure for MS. It is a patient-reported ordinal six-item measure whereby items are rated on a scale of 0 (not at all confident) to 100 (highly confident) and averaged to obtain a total score. All study outcomes will be measured at baseline and at months 3, 6, 9 and 12 in both study groups (Fig. 1). To avoid anticipation of surveys potentially influencing responses, participants will only be informed that they will receive survey requests at random times over the study period.

\section{Analyses}

Baseline data collection will include both demographic and MS-related information (Table 1); the same information will be collected at the end of the 12-month study period for all participants. All analyses will follow 
Table 1 Baseline data collection variables

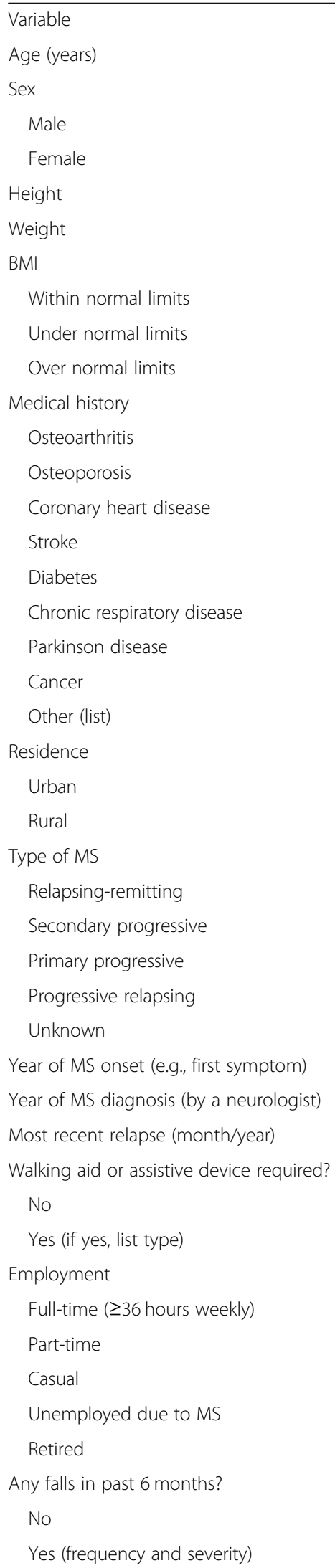

Table 1 Baseline data collection variables (Continued)

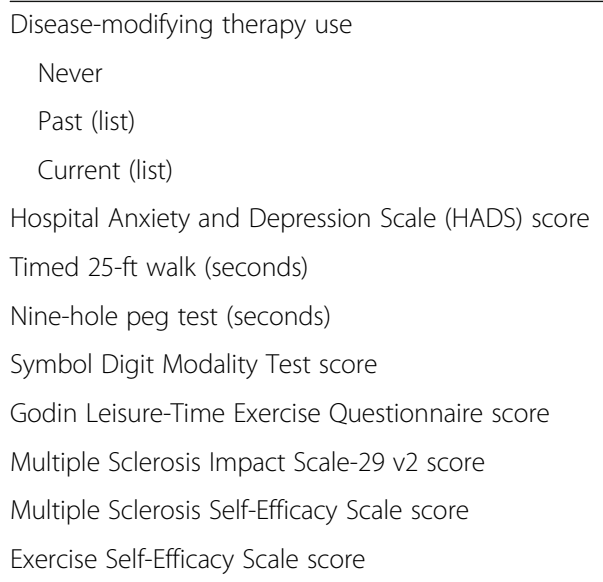

$B M I$ body mass index, MS multiple sclerosis

the intention-to-treat principle. The primary outcome will be analysed with repeated measures analysis of variance (ANOVA) and mixed effects models to compare GLTEQ scores between the intervention and control groups at 12 months. A mixed effects model will incorporate missing data under the assumption of missing at random. Although the GLTEQ is an ordinal scale, we will treat it as continuous because this is common practice for this measure [37] and will allow comparisons with the existing literature. Further within-subject effect comparison at 12 months will be done using ANOVA. The group-by-time interaction will be explored using a mixed effects model after controlling for potential covariates. Similar analyses will occur for the secondary outcomes.

Using a conservative effect size of 0.3 for the primary outcome (change in GLTEQ), a power of $80 \%$, and an alpha of 0.5 , we estimate that 120 participants are needed for this study, allowing for $20 \%$ dropout. All statistical analyses will be conducted by the study biostatistician with use of SAS software (SAS Institute Inc., Cary, NC, USA), and all study data will be managed in REDCap (Vanderbilt, v6.7).

\section{Monitoring}

This study, including the participant consent form, has received ethical approval from the University of Saskatchewan Biomedical Research Ethics Board. Because this is a low-risk intervention, no data monitoring review committee is required. However, the University of Saskatchewan Biomedical Research Ethics Board has the authority to audit the study at any time to ensure compliance with approved protocols. Monthly research meetings involving the research team will be held to discuss day-to-day management and organisation of the study, including participant recruitment, delivery of the intervention, and participant monitoring. Finally, a trial 
steering committee comprised of the principal investigators, co-investigators, patient and family advisors, funders, and other stakeholders will meet quarterly over the course of the study period to monitor the overall study conduct and progress.

\section{Dissemination}

Study results will be shared with all relevant end users by a variety of methods. Results will be shared with study participants and the public through community presentations (live or webinars) and social media. These communications will be facilitated by various stakeholders, including the Multiple Sclerosis Society of Canada Saskatchewan Division. Communication of study results will also be sent to policy and decision makers at the provincial health authority and government levels. Scientific publications and presentations will target researchers and healthcare professionals. A final de-identified dataset may be available from the researchers upon request.

\section{Discussion}

In 2016, the number of MS cases globally was estimated to be over 2.2 million [38]. North America has the highest number of reported cases of MS, with a prevalence of 165 per 100,000 [38]. MS places a significant burden on both individuals and society due to its disabling, long-term nature; high healthcare use; and lost productivity $[39,40]$. By 2031, MS-related healthcare expenses are projected to reach $\$ 2$ billion annually in Canada [41]. Physical activity interventions consistently show an improvement in walking function based on clinical trial data [6] and should be further evaluated as cost-effective methods in the management of MS [41, 42].

Individuals with MS report that it is challenging for them to engage in physical activity often enough to gain health benefits and have indicated the need for support to initiate and maintain physical activity [5]. Recognising that increasing and maintaining physical activity levels in MS can be challenging, we consulted with individuals with MS and their families/caregivers during the development of the study design, intervention, outcome measures, and dissemination plan to ensure relevance and feasibility for participants. The result is a novel study design that combines behaviour change strategies with expert (neurophysiotherapy) support to increase physical activity levels in individuals with MS.

As with any study, there are potential limitations to consider. First, our primary outcome is a self-reported measure and may be susceptible to recall bias. The value of patient-reported outcomes is recognised by many organisations, including the US Food and Drug Administration, as they not only measure specific outcomes but also capture an individual's perceptions of their health and experiences [43]. The use of patient-reported outcomes in MS research is also increasing [43, 44]. The GLTEQ is a validated measure that has been used extensively in MS research and only requires participants to recall the last 7 days of activity. We have also purposely not disclosed the timing of questionnaire distributions to participants to try to minimise any potential for the Hawthorne effect [45]. Because we are limiting our enrolment to ambulatory individuals with MS, the results will not be directly applicable to those who are nonambulatory. However, we have attempted to increase the study generalisability with our very limited exclusion criteria and by allowing the study intervention to be delivered in locations and via methods that are most convenient to the participants.

Physical activity has been identified as a top research priority by the MS community [46-48]. Our study is designed to be both feasible and replicable in real-world settings and may lead to new knowledge that could significantly impact the management and overall health of individuals with MS.

\section{Trial status}

Participant enrolment began on 19 July 2019. At the time of proof review (26 November 2019), enrolment is complete, and it is expected to be complete by December 2019.

\section{Supplementary information}

Supplementary information accompanies this paper at https://doi.org/10. 1186/s13063-019-3768-7.

Additional file 1. SPIRIT 2013 Checklist: Recommended items to address in a clinical trial protocol and related documents.

\section{Abbreviations}

ANOVA: Analysis of variance; BCTT: Behavior Change Technique Taxonomy; BMI: Body mass index; GLTEQ: Godin Leisure-Time Exercise Questionnaire; IPAC-MS: Individualized Physiotherapy and Activity Coaching for Multiple Sclerosis; MS: Multiple sclerosis; SMSDP: Saskatchewan MS Drugs Program

\section{Acknowledgements}

The authors gratefully acknowledge the following patient advisors for their invaluable role in the study development and for their continued involvement throughout the study period and dissemination of results: Bonnie Gleim, Cheryl Kadash, Debby Paquin, and Eugene Paquin. The authors also gratefully acknowledge Marla Fieber, Erin Kuan, and Dr. Gary Linassi, University of Saskatchewan, College of Medicine (Physical Medicine and Rehabilitation), for their support.

Authors' contributions

FLG and CDE drafted the manuscript. SJD and KBK obtained the study funding. SJD, KBK, CDE, and HJL designed the study protocol. All authors read and approved the final manuscript.

\section{Funding}

This study is supported by joint funding from the Saskatchewan Health Research Foundation and the Saskatchewan Centre for Patient-Oriented Research. The study sponsor had no role in the study design, writing of the manuscript, or decision to submit the manuscript for publication. 


\section{Availability of data and materials}

The de-identified dataset analysed during the current study may be available from the researchers on reasonable request.

\section{Ethics approval and consent to participate}

Informed consent will be obtained from all study participants prior to enrolment. This study, including the participant consent form, has received ethical approval from the University of Saskatchewan Biomedical Research Ethics Board (BIO-REB 1019).

\section{Consent for publication}

Not applicable; study protocol only.

\section{Competing interests}

FLG declares no competing interests. CDE receives research funding from the Canadian Institutes of Health Research and the Saskatchewan Health Research Foundation. KBK has received funding for the Saskatchewan MS Drugs Research Program from the Saskatchewan Ministry of Health. HJL declares no competing interests. MCL has received educational and/or consulting funding from Biogen Idec, Pendopharm, and Sanofi Genzyme. SJD receives research funding from the Saskatchewan Health Research Foundation and the Saskatchewan Centre for Patient-Oriented Research.

\section{Author details}

'College of Pharmacy and Nutrition, University of Saskatchewan, 104 Clinic Place, Saskatoon, SK, Canada. ${ }^{2}$ College of Medicine, University of Saskatchewan, 107 Wiggins Road, Saskatoon, SK, Canada.

\section{Received: 12 August 2019 Accepted: 29 September 2019}

Published online: 02 December 2019

\section{References}

1. Lublin FD, Reingold SC, Cohen JA, et al. Defining the clinical course of multiple sclerosis: the 2013 revisions. Neurology. 2014;83:278-86.

2. Confavreux C, Vukusic S. Natural history of multiple sclerosis: a unifying concept. Brain. 2006:129:606-16.

3. Confavreux C, Vukusic S, Adeleine P. Early clinical predictors and progression of irreversible disability in multiple sclerosis: an amnesic process. Brain. 2003: 126:770-82.

4. Freedman MS. Long term follow-up of clinical trials of multiple sclerosis therapies. Neurology. 2011;76:26-34

5. Hale LA, Smith C, Mulligan H, Treharne GJ. "Tell me what you want, what you really really want....": asking people with multiple sclerosis about enhancing their participation in physical activity. Disabil Rehabil. 2012;34: 1887-93.

6. Pearson M, Dieberg G, Smart N. Exercise as a therapy for improvement of walking ability in adults with multiple sclerosis: a meta-analysis. Arch Phys Med Rehabil. 2015;96:1339-48 e7.

7. Motl RW, Pilutti LA. Is physical exercise a multiple sclerosis disease modifying treatment? Expert Rev Neurother. 2016;16:951-60.

8. Kjølhede T, Vissing K, Dalgas U. Multiple sclerosis and progressive resistance training: a systematic review. Mult Scler J. 2012:18:1215-28.

9. Dalgas $U$, Stenager E. Exercise and disease progression in multiple sclerosis: can exercise slow down the progression of multiple sclerosis? Ther Adv Neurol Disord. 2012;5:81-95.

10. Motl RW, Learmonth YC, Pilutti LA, Gappmaier E, Coote S. Top 10 research questions related to physical activity and multiple sclerosis. Res Q Exerc Sport. 2015:86:117-29.

11. Kinnett-Hopkins D, Adamson B, Rougeau K, Motl RW. People with MS are less physically active than healthy controls but as active as those with other chronic diseases: an updated meta-analysis. Mult Scler Relat Disord. 2017;13: $38-43$.

12. Motl RW, Sandroff BM, Kwakkel G, et al. Exercise in patients with multiple sclerosis. Lancet Neurol. 2017;16:848-56.

13. Sangelaji B, Smith CM, Paul L, Sampath KK, Treharne GJ, Hale LA. The effectiveness of behaviour change interventions to increase physical activity participation in people with multiple sclerosis: a systematic review and meta-analysis. Clin Rehabil. 2015;30:559-76.

14. Michie S, Richardson M, Johnston $M$, et al. The Behavior Change Technique Taxonomy (v1) of 93 hierarchically clustered techniques: building an international consensus for the reporting of behavior change interventions. Ann Behav Med. 2013:46:81-95.

15. Bandura A. Health promotion by social cognitive means. Health Educ Behav. 2004;31:143-64.

16. Uszynski MK, Casey B, Hayes S, et al. Social cognitive theory correlates of physical activity in inactive adults with multiple sclerosis. Int J MS Care. 2018;20:129-35

17. Lai B, Young HJ, Bickel CS, Motl RW, Rimmer JH. Current trends in exercise intervention research, technology, and behavioral change strategies for people with disabilities: a scoping review. Am J Phys Med Rehabil. 2017;96:748-61.

18. Motl RW, Dlugonski D, Pilutti LA, Klaren RE. Does the effect of a physical activity behavioral intervention vary by characteristics of people with multiple sclerosis? Int J MS Care. 2015:17:65-72.

19. Stroud N, Minahan C, Sabapathy S. The perceived benefits and barriers to exercise participation in persons with multiple sclerosis. Disabil Rehabil. 2009;31:2216-22.

20. Streber R, Peters S, Pfeifer K. Systematic review of correlates and determinants of physical activity in persons with multiple sclerosis. Arch Phys Med Rehabil. 2016:97:633-45 e29.

21. Ploughman M. Breaking down the barriers to physical activity among people with multiple sclerosis - a narrative review. Phys Ther Rev. 2017;22:124-32

22. Stennett A, De Souza L, Norris M. Physical activity and exercise priorities in community dwelling people with multiple sclerosis: a Delphi study. Disabil Rehabil. 2018;40:1686-93.

23. Godin G. The Godin-Shephard Leisure-Time Physical Activity Questionnaire. Health Fitness I Canada. 2011:4:18-22

24. Amireault S, Godin G. The Godin-Shephard Leisure-Time Physical Activity Questionnaire: validity evidence supporting its use for classifying healthy adults into active and insufficiently active categories. Percept Mot Skills. 2015:120:604-22.

25. Sikes EM, Richardson EV, Cederberg KJ, Sasaki JE, Sandroff BM, Motl RW Use of the Godin Leisure-Time Exercise Questionnaire in multiple sclerosis research: a comprehensive narrative review. Disabil Rehabil. 2019:41:1243-67.

26. Hohol MJ, Orav EJ, Weiner HL. Disease steps in multiple sclerosis: a simple approach to evaluate disease progression. Neurology. 1995:45:251-5.

27. Warburton D, Jamnik V, Bredin SSD, Gledhill N. The ePARmed-X+ physician clearance follow-up. Health Fitness J Canada. 2014;7:35-8.

28. Michie S, van Stralen MM, West R. The behaviour change wheel: a new method for characterising and designing behaviour change interventions. Implement Sci. 2011;6:42

29. Multiple Sclerosis Society of Canada. Canadian physical activity guidelines for adults with multiple sclerosis. https://www.csep.ca/CMFiles/Guidelines/ specialpops/CSEP MS PAGuidelines adults en.pdf. Accessed 11 July 2019.

30. Multiple Sclerosis Society of Canada. Diet. https://mssociety.ca/hot-topics/ diet. Accessed 10 July 2019.

31. Multiple Sclerosis Society of Canada. Taming stress in multiple sclerosis. https://mssociety.ca/library/document/frgRLYhp2 oeZnMd0qWivQV8tAuwJPF5x/original.pdf. Accessed 11 July 2019.

32. Miller DJ, Freedson PS, Kline GM. Comparison of activity levels using the Caltrac $^{\oplus}$ accelerometer and five questionnaires. Med Sci Sports Exerc. 1994; 26:376-82.

33. Hobart J, Cano S. Improving the evaluation of therapeutic interventions in multiple sclerosis: the role of new psychometric methods. Health Technol Assess. 2009:13(12). https://doi.org/10.3310/hta13120.

34. Chiu CY, Motl RW. Further validation of the Multiple Sclerosis Self-Efficacy Scale. Disabil Rehabil. 2015:37:2429-38.

35. Schwartz CE, Coulthard-Morris L, Zeng Q, Retzlaff P. Measuring self-efficacy in people with multiple sclerosis: a validation study. Arch Phys Med Rehabil. 1996:77:394-8.

36. McAuley E. Self-efficacy and the maintenance of exercise participation in older adults. J Behav Med. 1993:16:103-13.

37. Johnson DR, Creech JC. Ordinal measures in multiple indicator models: a simulation study of categorization error. Am Sociol Rev. 1983;48:398-407

38. Wallin MT, Culpepper WJ, Nichols E, et al. Global, regional, and national burden of multiple sclerosis 1990-2016: a systematic analysis for the Global Burden of Disease Study 2016. Lancet Neurol. 2019:18:269-85.

39. Bergvall N, Lahoz R, Reynolds T, Korn JR. Healthcare resource use and relapses with fingolimod versus natalizumab for treating multiple sclerosis: a retrospective US claims database analysis. Curr Med Res Opin. 2014;30:1461-71. 
40. Karampampa K, Gustavsson A, Miltenburger C, Eckert B. Treatment experience, burden and unmet needs (TRIBUNE) in MS study: results from five European countries. Mult Scler J. 2012;18:7-15.

41. Amankwah N, Marrie RA, Bancej C, et al. Multiple sclerosis in Canada 2011 to 2031: results of a microsimulation modelling study of epidemiological and economic impacts. Health Promot Chronic Dis Prev Can. 2017;37:37-48.

42. Tosh J, Dixon S, Carter A, et al. Cost effectiveness of a pragmatic exercise intervention (EXIMS) for people with multiple sclerosis: economic evaluation of a randomised controlled trial. Mult Scler J. 2014;20:1123-30.

43. Khurana V, Sharma H, Afroz N, Callan A, Medin J. Patient-reported outcomes in multiple sclerosis: a systematic comparison of available measures. Eur J Neurol. 2017;24:1099-107.

44. Nowinski CJ, Miller DM, Cella D. Evolution of patient-reported outcomes and their role in multiple sclerosis clinical trials. Neurotherapeutics. 2017;14: 934-44.

45. McCarney R, Warner J, lliffe S, van Haselen R, Griffin M, Fisher P. The Hawthorne effect: a randomised, controlled trial. BMC Med Res Methodol. 2007;7:30

46. Donkers SJ, Smith C, Hale L, Milosavljevic S, Musselman K. Proposed solutions for improving physical activity from the perspective of persons living with multiple sclerosis in SK. Dallas: Poster presented at the American Congress of Rehabilitation Medicine Annual Conference; 2018.

47. Multiple Sclerosis Advisory Panel of Saskatchewan, Government of Saskatchewan. Multiple sclerosis advisory panel recommendations. 2016 http://publications.gov.sk.ca/documents/13/108828-MS-Advisory-PanelRecommendations.pdf. Accessed 10 July 2019.

48. Multiple Sclerosis Society of Canada. MS Wellness Survey Canada 2015. https://mssociety.ca/ms-wellness-survey. Accessed 11 July 2019.

\section{Publisher's Note}

Springer Nature remains neutral with regard to jurisdictional claims in published maps and institutional affiliations.

Ready to submit your research? Choose BMC and benefit from:

- fast, convenient online submission

- thorough peer review by experienced researchers in your field

- rapid publication on acceptance

- support for research data, including large and complex data types

- gold Open Access which fosters wider collaboration and increased citations

- maximum visibility for your research: over $100 \mathrm{M}$ website views per year

At $\mathrm{BMC}$, research is always in progress.

Learn more biomedcentral.com/submissions 